\title{
Derivation of Stochastic differential Equations for Scrape-off Layer Plasma fluctuations from experimentally measured statistics
}

\author{
A. Mekkaoui \\ Institute for Energy and Climate Research - Plasma Physics, \\ Research Center Jülich GmbH, Association FZJ-Euratom, D-52425 Jülich, Germany*
}

\begin{abstract}
A stochastic differential equation for intermittent plasma density dynamics in magnetic fusion edge plasma is derived, which is consistent with the experimentally measured gamma distribution and the theoretically expected quadratic nonlinearity. The plasma density is driven by a multiplicative Wiener process and evolves on the turbulence correlation time scale, while the linear growth is quadratically damped by the fluctuation level. The sensitivity of intermittency to the nonlinear dynamics is investigated by analyzing the nonlinear Langevin representation of the beta process, which leads to a root-square nonlinearity.
\end{abstract}

PACS numbers: 02.50.Ey, 52.65.Ff, 05.10.Gg, 52.25.Gj

The scrape-off layer (SOL) plasma of magnetic confinement fusion devices exhibits a universal intermittent behavior characterized by a strongly non-Gaussian statistics and spatiotemporal correlations [1]. The intermittent character of fluctuations is closely related to a bursty convective transport of over-density coherent structures (blobs) and edge localized mode filaments (ELMs) [2]. Basically, the deviation from normality is caused by the quadratically nonlinear turbulence, coupling the plasma density $(n)$ to the electric potential, i.e. $\partial_{t} n+\cdots \propto n n$ [3]. For instance, an assumed Gaussian initial condition $n_{0}$ evolves to a chi-square random variable (r.v.) $\left(n_{0}^{2}\right)$ and so forth. The closure procedure reducing the coupled deterministic turbulence equations to a nonlinear Langevin equation is still a challenging problem [4]. An interpretation attempt of the dynamical origin of intermittency and its statistical signature has been proposed [5]. It describes the intermittent quantity $n$ as a quadratic polynomial of a Gaussian variable $g$, i.e.,

$$
n(t)=g(t)+\Omega g^{2}(t)
$$

where $\Omega$ is the non-normality parameter which measures the deviation from the Gaussian statistics and the strength of the nonlinear coupling. It is not surprising that the process Eq. (1) satisfies the universal parabolic relation between the Kurtosis and the Skewness, i.e., $K=a_{0} S^{2}+a_{1}$, observed in several turbulent media [4], because the variable $n+1 /(4 \Omega)$ is distributed as $\Omega(g+1 /(2 \Omega))^{2}$. This is the non-central chi-square distribution by construction, which is a particular case of the gamma distribution of density fluctuations often measured in the edge of fusion devices and satisfying $a_{0}=1.5$ and $a_{1}=3[6,7]$. However, the physical mechanism behind Eq. (1) is still not clear and the Gaussianity assumption of the dynamical variable $g$ appears to be too strong.

Before deriving the stochastic differential equation for

*s.mekkaoui@fz-juelich.de edge plasma turbulence, it is worth briefly reviewing some existing stochastic models which could explain the emergence of the gamma statistics from a turbulent plasma. A correspondance and closer similarities between them are also pointed out in this Letter. The most important result overlooked in contemporary description of edge plasma turbulence work is due to Jakeman and Pusey [8]. In their investigation of radiation scattering through a fluctuating background, they showed that the gamma probability distribution function (PDF) appears as a limit distribution of a finite sum of independent and identically distributed random perturbations $x_{i}$,

$$
n(t)=\sum_{i=1}^{N(t)} x_{i},
$$

where their number $N(t)$ obeys a birth-deathimmigration process with respective rates $b, d$ and $m$ [8]. Without any condition on the perturbers distribution $P\left(x_{i}\right)$, and when the death rate is close to the birth rate $(b \simeq d)$, then $n$ is gamma distributed with scale factor $\mu=\langle x\rangle$ and shape factor $\nu=m / b$,

$$
P(n)=\frac{n^{\nu-1}}{\mu^{\nu} \Gamma(\nu)} \exp (-n / \mu) .
$$

On the other hand, in Ref. [9] the gamma distribution is derived by applying Campbell's theorem [10] to the plasma density signals, assumed to be a linear superposition of $N$ bursts, i.e.,

$$
n(t)=\sum_{i=1}^{N} x_{i} \mathcal{F}\left(t-t_{i}\right),
$$

with exponentially distributed intensity $P(x)=$ $1 / \mu \exp (-x / \mu)$, waiting time between bursts arrivals $P(t)=\exp \left(-t / \tau_{w}\right)$ and burst life time $\mathcal{F}(t)=$ $\exp \left(-t / \tau_{d}\right)$. Beyond their structural similarity, it is noteworthy that both stochastic processes given by Eq. (2) and Eq. (4) lead exactly to the same PDF Eq. (3), when the waiting time and the duration time are given by $\tau_{w}=$ 
$m^{-1}$ and $\tau_{d}=b^{-1} \simeq d^{-1}$, respectively. The equivalence between both derivations of the gamma statistics has a physical meaning, since we could make correspondence between the immigration process and the waiting time, and between the birth process and the duration time. The similarities between these two gamma processes is extended beyond the univariate statistics by investigating their temporal correlation. The covariance function of the process Eq. (4) is calculated using random noise properties [10], $C(t)=\left(\tau_{w} / \tau_{d}\right) \mu^{2} \exp \left(-t / \tau_{d}\right)$, showing that the correlations are introduced only through a single burst duration, since bursts are independent. The correlation structure of the shot noise process Eq. (4) is consistent with that of Eq. (2), which is also exponential with the inverse death rate as a correlation time $C(t) \propto \exp (-b t)[11]$.

Although the model Eq. (4) is consistent with the experimental measurements, it obeys a linear stochastic differential equation [12], which is surprising because the intermittent statistics in turbulent plasma is often associated to nonlinear turbulence models like the HasegawaWakatani system equations [3]. Indeed the stochastic models Eq. (2) and Eq. (4) could explain the observed gamma statistics from the response function of the instrumental devices point of view e.g., Langmuir probe, but their dynamical content is still far from the theoretical predictions. Finally, note that we have introduced a multivariate gamma distribution to account for the effect of plasma density fluctuations on the transport of neutral particles [13]. This is a descriptive approach, which does not aim at explaining why the fluctuations are gamma distributed.

In this Letter, we provide a bridge between the nonlinear dynamics and the intermittent statistics in edge plasma of magnetic fusion devices. Unlike existing work, here the nonlinear dynamics is investigated starting from the experimentally measured distribution. Namely, we assume gamma statistics together with a quadratic nonlinearity as a theoretical constraint to derive a plasma turbulence equation. In addition to the measured PDF, the link to experiments is reinforced by introducing the correlation time and the fluctuations amplitude of turbulence in the model. These quantities are commonly measured in edge plasma experiments. They are respectively given by $\tau_{c}=\int_{0}^{\infty}|\rho(t)| d t$ and $R=\left\langle\tilde{n}^{2}\right\rangle^{1 / 2} /\langle n\rangle$, where $\tilde{n}=n-\langle n\rangle$, and $\rho(t)$ the correlation function,

$$
\rho(t)=\left\langle\tilde{n}\left(t+t^{\prime}\right) \tilde{n}\left(t^{\prime}\right)\right\rangle /\left\langle\tilde{n}^{2}\right\rangle .
$$

Usually $\langle\ldots\rangle$ denotes to the time average over a range larger than the turbulence correlation time, $\langle f[n]\rangle=$ $(1 / T) \int_{0}^{T} f\left[n\left(t^{\prime}\right)\right] d t^{\prime}$, which is equivalent to the statistical averaging $\langle\cdots\rangle_{s}=\int P(n) f(n) d n$, when $T \rightarrow \infty$ in virtue of the ergodic theorem.

The gamma PDF Eq. (3) is a special case of the Pearson distributions family (Pearson type-III) [14]. All these distributions obey the differential equation $\partial_{n} P(n)=$ $F(n) P(n) / H(n)$, called the Pearson equation in the fol- lowing. The coefficients in the polynomials $F=n+u$ and $H=v_{0}+v_{1} n+v_{2} n^{2}$ are entirely expressed in terms of the first four moments $m_{k+1}=\int n^{k+1} P(n) d n$, by solving the system equations $\int n^{k} H(n) \partial_{n} P(n) d n=$ $\int n^{k} F(n) P(n) d n$, for $k=0, \ldots, 3$. This statement makes the Pearson equation very useful for the statistical characterization of edge plasma turbulence, since the first four moments of turbulence play a central role through the fluctuations amplitude $R$ and the $K-S$ scaling. The associated stochastic processes are specified by the following Fokker-Planck equation for $P(n, t)[15,16]$,

$$
\frac{\partial P}{\partial t}=-\frac{\partial}{\partial n}\left[\left(F+\frac{\partial H}{\partial n}\right) P\right]+\frac{1}{2} \frac{\partial^{2}}{\partial n^{2}}(2 H P) .
$$

We emphasize here the non uniqueness of the derived Fokker-Planck equation, because of the occurence of the ratio $F(n) / H(n)$ in the Pearson equation. This property permits us to change the dynamics of $n$ without modifying its statistics through the multiplication of $P$ and $H$ by the same function. One finds the irreducible Pearson representation of the gamma distribution Eq. (3),

$$
\frac{\partial P(n)}{\partial n}=\frac{(1-1 / \nu)\langle n\rangle-n}{\langle n\rangle n / \nu} P(n),
$$

where $\nu=R^{-2}$ and $\langle n\rangle=\nu \mu$. Using Eq. (6), we derive the corresponding Fokker-Planck equation,

$$
\frac{\partial P}{\partial t}=-\frac{\partial}{\partial n}\left[\frac{1}{\tau}(\langle n\rangle-n) P\right]+\frac{1}{2} \frac{\partial^{2}}{\partial n^{2}}[2\langle n\rangle n P / \nu \tau],
$$

where we have used the degeneracy of the Pearson equation to introduce a characteristic time scale $\tau$ which is specified later. Then, using the Feynman-Kac formula [12], to identify the coefficients of the corresponding Langevin equation,

$$
d n(t)=(\langle n\rangle-n(t)) \frac{d t}{\tau}+\sqrt{2\langle n\rangle n(t) / \nu \tau} d w(t),
$$

where $d w=\eta \sqrt{d t}$ is the Wiener process (Brownian motion) and $\eta$ is normally distributed with mean 0 and variance 1. The stochastic model Eq. (9) has a root-square nonlinearity because of the term $\propto \sqrt{n}$ and is broadly used in financial forecasting [17]. This is not consistent with the quadratically nonlinear structure of turbulence equations [3]. However, as previously noticed, Eq. (7) is degenerate and allows us to derive a higher order nonlinear stochastic differential equation. Multiplying the denominator and the numerator on the right hand side of Eq. (7) by $n \tau$, we obtain a new Pearson representation of the gamma process. Then the corresponding stochastic differential equation follows from Eq. (6),

$$
d n=\gamma n(t) d t-\frac{n^{2}(t)}{\langle n\rangle \tau} d t+R \sqrt{2 / \tau} n(t) d w(t),
$$

with $\gamma=\left(1+R^{2}\right) / \tau$. Equation (10) is the main result of this Letter, it is quadratically nonlinear and has the 


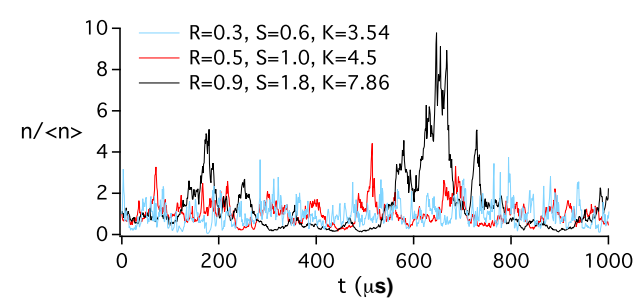

FIG. 1. a quiet (blue), intermittent (red) and strongly intermittent (black) time series of the plasma density normalized to its local average value.

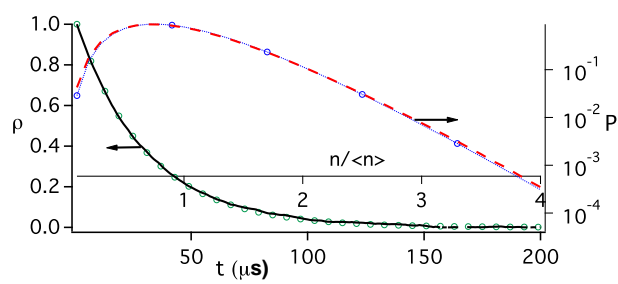

FIG. 2. the input gamma PDF in semilogarithmic scale (blue) and the analytical derived correlation function $\exp \left(-t / \tau_{c}\right)$ (black), showing a good agreement with the numerical PDF (red) and correlation (green) constructed from the time series for $R=50 \%$ and $\tau_{c}=30 \mu \mathrm{s}$.

gamma distribution as a stationary solution. The process Eq. (10) has a clear physical interpretation. The linear growth is driven by the fluctuations amplitude. As a response to this growth, a nonlinear damping takes place, which is proportional to the fluctuation level $n(t) /\langle n\rangle$. The dynamics is driven by a multiplicative white noise of variance $R^{2}$. Unlike Eq. (1) which is based on the Gaussian assumption of the dynamical variable, and the process Eq. (4) obeying a linear stochastic differential equation, the stochastic model Eq. (10), shows how the gamma distribution and the associated universal $K-S$ scaling arise from the quadratically nonlinear dynamics of the intermittent variable. Equation (10) takes the form of the L-H transition paradigm equation, e.g. [18], with the difference that the latter is quadratic for the energy $E=((n-\langle n\rangle) /\langle n\rangle)^{2}$.

In order to clarify the role of the time scale $\tau$, we study the dynamics of the correlation function $\rho(t)$. Integrating Eq. (10) over $\left[t^{\prime}, t+t^{\prime}\right]$, multiplying both sides by $n\left(t^{\prime}\right)$, averaging over $t^{\prime}$, canceling the term $\propto\langle d w\rangle$ and taking the time derivative we obtain

$$
\partial_{t} \rho(t)-\gamma \rho(t)=\frac{\gamma}{R^{2}}-\frac{1}{\tau R^{2}\langle n\rangle^{3}}\left\langle n^{2}\left(t+t^{\prime}\right) n\left(t^{\prime}\right)\right\rangle .
$$

The term between brackets is a two-time correlation and can be approximated by,

$$
\left\langle n^{2}\left(t+t^{\prime}\right) n\left(t^{\prime}\right)\right\rangle=\theta \rho(t)+\kappa
$$

The constant coefficients are fixed using the two known limits, $\tau_{c}=0, \rho(t)=0$ and $\tau_{c}=\infty, \rho(t)=1$, leading to $\kappa=\langle n\rangle\left\langle n^{2}\right\rangle$ and $\theta=\left\langle n^{3}\right\rangle-\langle n\rangle\left\langle n^{2}\right\rangle$. Then Eq. (11) reduces to $\partial_{t} \rho(t)+\gamma \rho(t)=0$, showing that the correlation function is exponential, i.e., $\rho(t)=\exp (-\gamma t)$. Therfore, $\tau_{c}=\tau /\left(1+R^{2}\right)$ is nothing else but the correlation time of turbulent fluctuations. It is worth noticing that the approximation Eq. (12) becomes exact when the process Eq. (10) reaches its stationnary distribution starting with arbitrary initial condition. The proof of this statement is trivial for half integer shape factor $\nu=I / 2$, with $I$ the degree of freedom of the gamma distribution. In this case the gamma r.v. is expressed as the sum of gaussian r.v.s [19], $n(t)=\sum_{k=1}^{I} g_{k}^{2}(t)$ with $\langle g\rangle=0$ and

$$
\left\langle g_{k}\left(t+t^{\prime}\right) g_{l}\left(t^{\prime}\right)\right\rangle=\left\langle g^{2}\right\rangle(\rho(t))^{1 / 2} \delta_{k l},
$$

where $\rho$ is given by Eq. (5). Without loss of generality, we use the Wick theorem for the particular case $I=1$ to develop the left hand side in Eq. (12),

$$
\left\langle g^{4}\left(t+t^{\prime}\right) g^{2}\left(t^{\prime}\right)\right\rangle=12\left\langle g^{2}\right\rangle\left\langle g\left(t+t^{\prime}\right) g\left(t^{\prime}\right)\right\rangle^{2}+3\left\langle g^{2}\right\rangle^{3},
$$

then using the identity Eq. (13) in the right hand side of Eq. (14) to recover Eq. (12). A generalization of the result to arbitrary $\nu$ is achieved by assuming a Markov correlation function, i.e., $\rho\left(t-t^{\prime}\right)=\rho\left(t-t^{\prime \prime}\right) \rho\left(t^{\prime \prime}-t^{\prime}\right)$ with $t>t^{\prime \prime}>t^{\prime}$, which allows us to derive Eq. (12) from the multivariate characteristic function [20]. The Markov assumption is then validated since the resulting correlation is exponential.

In order to generate intermittent plasma density time series from Eq. (10), we proceed as following. The time series is initiated by fixing a positive and arbitrary initial condition $n(0)$, sampling independent normally distributed r.v. $\eta$, and writing $d w(t)=\eta \sqrt{d t}$ in the right hand side of Eq. (10). The time series is then constructed by iteration using $d n=n(t+d t)-n(t)$. In Fig. 1 are plotted three time series with a typical Langmuir probe resolution $d t=1 \mu \mathrm{s}$ as a time step. The blue curve represents a quiet time series with $R=30 \%$ and $\tau_{c}=3 \mu \mathrm{s}$, as in the typical edge plasma conditions close to the LCFS [6]. The red curve is representative of an intermediate situation corresponding to the near SOL with moderate fluctuations amplitude $R=50 \%$ and $\tau_{c}=16 \mu \mathrm{s}$, one burst exceeding the average value by a factor of 4 arises in the range of $1 \mathrm{~ms}$. A strongly intermittent time series is illustrated by the black curve, with $R=90 \%$ and $\tau_{c}=55$ $\mu \mathrm{s}$. Three bursts exceeding $4\langle n\rangle$ and one "super burst" with the amplitude of $10\langle n\rangle$ are observed, as is typically the case in the far SOL of tokamaks [7]. In Fig. (2) is plotted the input gamma PDF Eq. (3) (blue curve) which is in good agreement with the output distribution (red curve) contructed from the time series Eq. (10), showing the consistency of the stochastic model. A numerical testing of the derived correlation function (black curve) is also presented in Fig. (2), showing a good agreement with the correlation contructed from the time series (green curve). A simple diagnostic allows one to check whether measurements are consistent with Eq. (10). It consists in 
using experimental time series $n(t)$ to take out the multiplicative noise from Eq. (10). If the PDF of this noise is Gaussian then, the time series is both gamma distributed and sustained by a quadratically nonlinear dynamics.

In order to investigate the sensitivity of the intermittent fluctuations statistics to its underlying nonlinear dynamics, we compare the gamma process to other proposed stochastic models. According to its capability to have negative skewness, the beta distribution has been used to fit the plasma density data in the edge of TORPEX [7]. Let us consider here the standardized beta distribution, $P(n) \propto n^{\alpha-1}(1-n)^{\beta-1}, 0<n<1, \alpha, \beta>$ 0 , where $\alpha=1 / R^{2}-\langle n\rangle\left(1+1 / R^{2}\right)$ and $\beta=\alpha(1 /\langle n\rangle-1)$. The beta distribution obeys the Pearson equation, then we proceed as for the gamma distribution to derive the following stochastic differential equation, $d n=(\alpha+$ 1) $n d t / \tau+(\beta-\alpha-3) n^{2} d t / \tau+n \sqrt{2(1-n) / \tau} d w(t)$, showing that the quadratically nonlinear dynamics of the beta process is systematically accompanied with a root-square nonlinearity. The comparison between the gamma and the beta processes shows the importance of the PDF functional form used to fit experimental data. With identical two first moments, different distributions (gamma, beta and log-normal) could provide a reasonable fit of experimental measurements, although their underlying dynamics is different. Therefore, and in order to improve the connection between the theory of nonlinear dynamics and experimental time series, the analysis of plasma's fluctuations using strong criteria are suitable to clarify whether the time series statistics follows a given distribution. It is preferable to the data fitting and a statistical signature based on the $K-S$ scaling, since no unique correspondence exists between this scaling and the un-

[1] B. A. Carreras, J. Nucl. Mater. 337, 315 (2005).

[2] D. A. D'Ippolito, J. R. Myra, and S. J. Zweben, Phys. Plasmas 18, 060501 (2011).

[3] J. A. Krommes, Phys. Report 360, 1 (2002).

[4] J. A. Krommes, Phys. Plasmas 15, 030703 (2008).

[5] I. Sandberg, S. Benkadda, X. Garbet, G. Ropokis, K. Hizanidis, and del-Castillo-Negrete, Phys. Rev. Lett. 103, 165001 (2009).

[6] J. P. Graves, J. Horacek, R. A. Pitts, and K. I. Hopcraft, Plasma Phys. Controlled Fusion 47, L1 (2005).

[7] B. Labit, I. Furno, A. Fasoli, A. Diallo, S. H. Müller, G. Plyushchev, M. Podest, and F. M. Poli, Phys. Rev. Lett. 98, 255002 (2007).

[8] E. Jakeman and P. N. Pusey, Phys. Rev. Lett. 40, 546 (1978).

[9] O. E. Garcia, Phys. Rev. Lett. 108, 265001 (2012).

[10] S. O. Rice, Bell Syst. Tech. J. 23, 282 (1944).

[11] E. Jakeman, J. Phys. A: Math. Gen. 13, 31 (1980).

[12] C. Gardiner, Stochastic methods (Springer, 2009).

[13] A. Mekkaoui, Y. Marandet, D. Reiter, P. Boerner, P. Genesio, J. Rosato, R. Stamm, H. Capes, M. Koubiti, and L. Godbert-Mouret, Phys. Plasmas 19, 060701 derlying distributions. An interesting strong statistical discrimination criteria is given in [21]. It shows that if for independent random variables $y$ and $z$, then their sum $s=y+z$ and their ratio $r=y / z$ are independent if and only if both $y$ and $z$ are gamma distributed with the same scale factor. Others criteria can be found in $[22,23]$. In practice, the r.v.s $n(t)$ and $n(t+\Delta T)$ are selected into the same time series. The time separation $\Delta T$ should be large enough such that $n(t)$ and $n(t+\Delta T)$ are independent. The stochastic model developped in this Letter can be used to generate time series that can serve as a test for strong criteria. In particular, the time separation $\Delta T$ might be estimated from the stochastic model and used in experimental time series.

In summary, we have derived a stochastic differential equation for the intermittent plasma density in the SOL of fusion plasma devices. The plasma density dynamics evolves on a turbulence correlation time scale $\tau_{c}$, and is characterized by the local fluctuations amplitude $R$. This equation is consistent with both experimental measurements and theoretical predictions, since it has the gamma distribution as a stationary solution and follows a quadratic nonlinearity. A satisfactory description of edge turbulent transport requires space dependent equations for both the plasma density and the electric potential $(\phi)$ as coupled fields. The result developped in this Letter is a first step towards a more sophisticated stochastic models based on both experimental measurements and theoretical predictions. A stochastic differential equations of the form $d n=\mathcal{N}(n(x, t), \phi(x, t))+n d w$, appears promising to reach this goal. A deep analysis of spatiotemporal experimental time series, using strong criteria, could help us to focus on the relevant candidate distribution and to approach the nonlinear structure of $\mathcal{N}$. A future work is dedicated to this aim.
(2012).

[14] H. Cramér, Mathematical Methods of Statistics (Asia Publishing House, 1961).

[15] T. Ozaki, Nonlinear time series models and dynamical systems, in Handbook of Statistics, Vol. 5 (NorthHolland, Amsterdam, Elsevier Science Publishers, 1985).

[16] T. Ozaki, Statistica Sinica 2, 113 (1992).

[17] J. C. Cox, J. E. Ingersoll, and J. S. A. Ross, Econometrica 53, 385 (1985).

[18] N. H. Bian, Phys. Plasmas 17, 044501 (2010).

[19] P. Krishnaiah and M. Rao, The American Mathematical Monthly 68, 342 (1961).

[20] S. Kotz, N. Balakrishnan, and N. L. Johnson, Continuous Multivariate Distributions (John Wiley \& Sons, Inc., 2000).

[21] E. Lukacs, Ann. Math. Stat. 26, 319 (1955).

[22] E. McKenzie, J. Appl. Prob. 19, 463 (1982).

[23] A. M. Kagan, Y. V. Linnik, and C. R. Rao, Characterization Problems in Mathematical Statistics (John Wiley \& Sons, New York, 1973). 